\section{Prevalência de diabetes mellitus e identificação de fatores associados em adultos residentes em área urbana de Ribeirão Preto, São Paulo, Brasil, 2006: Projeto OBEDIARP}

\author{
Diabetes mellitus prevalence and associated factors \\ in adults in Ribeirão Preto, São Paulo, Brazil, 2006: \\ OBEDIARP Project
}

\author{
${ }^{1}$ Escola de Enfermagem de \\ Ribeirão Preto, Universidade \\ de São Paulo, Ribeirão Preto, \\ Brasil. \\ 2 Departamento de Medicina \\ Preventiva, Universidade \\ Federal de São Paulo, \\ São Paulo, Brasil. \\ 3 Instituto de Economia \\ Agrícola, São Paulo, Brasil. \\ Correspondência \\ S. A. Moraes \\ Escola de Enfermagem de \\ Ribeirão Preto, Universidade \\ de São Paulo. \\ Av. Bandeirantes 3900 \\ Ribeirão Preto, $S P$ \\ 14040-902, Brasil. \\ samoraes@usp.br
}

\begin{abstract}
To identify diabetes mellitus prevalence and associated factors, a cross-sectional epidemiological study was developed including participants aged 30 years and older living in Ribeirão Preto, São Paulo State, Brazil. Using three-stage cluster sampling, probability weights were applied, resulting in a weighted sample of 2,197 participants. Diabetes mellitus diagnosis was based on previous medical history or World Health Organization (WHO) cut-offs after oral glucose tolerance test. To investigate associated factors, crude and adjusted prevalence ratios were estimated by points and confidence intervals, using Poisson regression. Diabetes mellitus prevalence was 15.02\%. After adjusting for potential confounding, factors associated with diabetes mellitus in the final model were: age; family history of diabetes mellitus; waist hip ratio; waist height ratio; number of medicines taken; and use of outpatient services. The results showed high diabetes mellitus prevalence and identified associated factors amenable to intervention.
\end{abstract}

Diabetes Mellitus; Adult Health; Risk Factors
Suzana Alves de Moraes 1 Isabel Cristina Martins de Freitas 1 Suely Godoy Agostinho Gimeno 2 Lenise Mondini ${ }^{3}$

\section{Introdução}

Considerando-se o atual estágio da transição demográfico-epidemiológica, o diabetes mellitus, ao lado de outros desfechos crônicos, é considerado um problema de saúde pública, não apenas em países desenvolvidos, como também em países em desenvolvimento.

Em 1998, King et al. ${ }^{1}$ projetaram um aumento de $35 \%$ na prevalência de diabetes mellitus, no mundo, passando de $4 \%$, em 1995 , para $5,4 \%$, em 2025. O crescimento relativo da prevalência será, entre 1995 e 2025, da ordem de $48 \%$ para os países em desenvolvimento, contra $27 \%$ para os países desenvolvidos.

Em 2004, Wild et al. ${ }^{2}$ publicaram resultados de estudo desenvolvido pela Organização Mundial da Saúde (OMS), referente às estimativas de prevalência do diabetes mellitus entre 2000 e 2030. Segundo os autores, o Brasil, que em 2000 ocupava o oitavo lugar entre os dez países com maior número de casos de diabetes (4,6 milhões), ocupará a sexta posição em 2030, quando contará com 8,9 milhões de pessoas diagnosticadas.

Para Narayan et al. 3 , mudanças na susceptibilidade genética para a doença não são os únicos fatores responsáveis pelo aumento da prevalência, sendo incriminados, principalmente, os fatores ambientais. Davies et al. 4 advogam, por outro lado, que mais de $75 \%$ das pessoas com diabetes mellitus tipo II morrem por complicações macrovasculares e, que sua esperança de 
vida pode ser encurtada em até 15 anos. Estes autores também referem que as melhores evidências para a prevenção do diabetes mellitus constituem-se de intervenções sobre o estilo de vida (prática de atividade física e mudanças no padrão dietético) tendo, como alvo, populações em maior risco. A relevância de intervenções sobre o estilo de vida, em indivíduos portadores de tolerância diminuída à glicose e glicemia de jejum alterada foi também reconhecida por Unwin et al. ${ }^{5}$, em estudo de revisão.

No Brasil, não existem informações sistemáticas sobre a prevalência de desfechos crônicos ou de seus fatores de risco/proteção, o que tem dificultado a formulação de hipóteses, ainda que em base agregada, sobre a influência de determinados fatores sobre estes desfechos.

Em relação aos estudos de prevalência, merece destaque por sua abrangência, o Censo de Diabetes, conduzido entre 1986 e 1988 em nove capitais brasileiras. Naquela ocasião, Malerbi \& Franco ${ }^{6}$ relataram que a prevalência de diabetes mellitus no Brasil foi 7,4\% (prevalência ajustada por idade). No mesmo estudo, os autores detectaram prevalência de 9,7\% para o Município de São Paulo que apresentou a maior magnitude do indicador entre as nove capitais avaliadas.

Rosa et al. 7, em estudo conduzido com dados do Sistema de Informações Hospitalares do Sistema Único de Saúde (SIH/SUS), no período entre 1999 e 2001, estimaram 327.800 internações por diabetes mellitus como diagnóstico principal (códigos E-10 a E-14 da 10a revisão da Classificação Internacional de Doenças - CID10). A Região Sudeste se destacou por apresentar maior número de óbitos hospitalares/1.000.000 habitantes, a partir dos 20 anos de idade (sexo masculino) e letalidade hospitalar de 13,3\%, em indivíduos do sexo masculino com 75 anos e mais. O custo médio por internação foi equivalente a US\$150,59 (intervalo de 95\% de confiança - IC95\%: 149,87-151,32), sendo maior no sexo masculino (US\$155,36) que no feminino (US\$147,67). Segundo Barceló et al. 8, as hospitalizações por diabetes representam $10 \%$ dos custos diretos com diabetes mellitus na América Latina.

Em relação às complicações do diabetes mellitus, resultados recentes de estudos de coorte têm evidenciado ou confirmado elevado risco de isquemia miocárdica, acidentes vasculares cerebrais e doença arterial periférica, em diabéticos, comparados com não diabéticos, mesmo que o diabetes não esteja associado com hipertensão arterial 9,10,11. Zhang et al. 12 apresentando resultados do National Health and Nutrition Examination Survey (NHANES, 1999-2004), revelaram que o diabetes mellitus está associado com a deficiência visual, não passível de correção, mesmo após o ajustamento para variáveis sócio-demográficas como idade, raça, nível de educação, renda e acesso a seguro-saúde (odds ratio - OR = 1,49; IC95\%: 1,01-2,20).

Levando-se em consideração a relevância do diabetes mellitus no âmbito da saúde pública, bem como a escassez, no Brasil, de estudos epidemiológicos analíticos, de base populacional, o presente estudo teve por objetivo investigar a prevalência de diabetes mellitus e fatores associados, na população urbana com 30 anos e mais, residente no Município de Ribeirão Preto, São Paulo, em 2006.

\section{Material e métodos}

O estudo apresenta delineamento transversal 13, sendo parte integrante do Projeto OBEDIARP, estudo epidemiológico de base populacional, tendo como um de seus objetivos a investigação da prevalência de diabetes mellitus e fatores associados, no Município de Ribeirão Preto. O Projeto OBEDIARP, incluindo todas as etapas do plano amostral, identificação de elegíveis, treinamento dos entrevistadores e coleta de dados foi conduzido no período compreendido entre fevereiro de 2005 e junho de 2007. A população do estudo foi composta por adultos com 30 anos e mais, de ambos os sexos, residentes em área urbana do Município de Ribeirão Preto.

O processo de amostragem foi desenvolvido em três estágios e, a precisão das estimativas, calculadas em amostra de 1.205 indivíduos, correspondeu a erros de amostragem fixados em torno de $2 \%$ (para prevalências abaixo de $15 \%$ ou acima de $75 \%$ ) e em torno de $3 \%$ (para prevalências entre $20 \%$ e $80 \%$ ). No primeiro estágio, 81 setores censitários foram sorteados, e, na seqüencia, 1.671 domicílios e 1.205 participantes foram sorteados, correspondendo, respectivamente, ao segundo e terceiro estágios de amostragem. O setor censitário foi a Unidade Primária de Amostragem (Instituto Brasileiro de Geografia e Estatística. Censo demográfico 2000. http://www.ibge.gov.br), e o domicílio e as pessoas, as unidades sorteadas no segundo e terceiro estágios, respectivamente. O método de sorteio por conglomerados, sob partilha proporcional ao tamanho, foi adotado nos dois primeiros estágios. No terceiro estágio, sorteou-se uma pessoa, com 30 anos ou mais, entre os (N) residentes nos domicílios sorteados. No segundo estágio de sorteio, estratificação por renda nominal média do chefe da família foi introduzida. No terceiro estágio, a estratificação levou em consideração a composição por sexo e faixa etária 14 . Ao todo, 930 participantes de am- 
bos os sexos foram entrevistados. A variabilidade introduzida, principalmente na terceira fração de amostragem, foi corrigida por meio do cálculo de pesos amostrais que levaram em consideração o "número de unidades elegíveis de cada domicílio" e as taxas de "não resposta", em cada setor censitário, originando uma amostra ponderada (nw) de 2.197 participantes com 30 anos e mais, residentes na área urbana do município ${ }^{14}$. Os pesos amostrais $\left(\mathrm{w}_{12}\right)$ foram calculados a partir do produto do inverso das frações amostrais $w_{1}$ e $\mathrm{w}_{2}$, onde:

$\mathrm{w}_{1}=\underline{\text { número de sorteados }} \mathrm{e}$; número de elegíveis

$\mathrm{w}_{2}=\underline{\text { número de entrevistados }}$ número de sorteados

No terceiro estágio de sorteio do Projeto OBEDIARP (sorteio dos participantes), foram excluídas mulheres gestantes ou puérperas, até o sexto mês após o parto. A taxa de resposta do Projeto OBEDIARP foi $78 \%$.

\section{Variáveis do estudo}

\section{- Classes de intolerância à glicose: diabetes mellitus, tolerância diminuída à glicose e glicemia de jejum alterada}

Após 12 horas de jejum, todos os participantes foram submetidos a dosagens de glicemias que foram aferidas em amostras de sangue capilar, coletadas em tiras-teste $(\mathrm{mg} / \mathrm{dL})$, após digitopunctura com lancetadores automáticos (Roche Diagnostics GmbH, Mannheim, Alemanha), sendo a leitura efetuada pelo método de colorimetria por reflectância, em glicosímetros portáteis da marca Accutrend (Roche Diagnostics GmbH, Mannheim, Alemanha). Os participantes, sem diagnóstico prévio de diabetes e com glicemias de jejum abaixo de $200 \mathrm{mg} / \mathrm{dL}$, foram também submetidos a estas dosagens, duas horas após sobrecarga oral com solução padronizada, contendo $75 \mathrm{~g}$ de glicose pura. O tempo de ingestão foi padronizado em cinco minutos. Os pontos de corte para a classificação de diabetes mellitus e das outras classes de intolerância à glicose (tolerância diminuída à glicose e glicemia de jejum alterada), foram definidos segundo recomendações da OMS 15 para aferições em sangue capilar. Os participantes foram classificados como "normais", desde que não referissem história prévia de diabetes mellitus e cujas dosagens de glicemia de jejum e pós-sobrecarga apresentassem, respectivamente, valores $<100 \mathrm{mg} / \mathrm{dL}$ ou $<140 \mathrm{mg} / \mathrm{dL}$. Aqueles com história prévia de diabetes, ou com glicemias de jejum em sangue capilar $\geq 200 \mathrm{mg} / \mathrm{dL}$ foram classificados como diabéticos. Os participantes foram classificados como pertencentes à classe glicemia de jejum alterada, desde que não referissem história prévia de diabetes, apresentassem glicemias de jejum $\geq 100 \mathrm{mg} / \mathrm{dL}$ e $<110 \mathrm{mg} / \mathrm{dL}$ e glicemia pós-sobrecarga $<140 \mathrm{mg} / \mathrm{dL}$. Na classe tolerância diminuída à glicose foram incluídos os participantes que, sem história prévia de diabetes, apresentassem glicemias de jejum $<110 \mathrm{mg} / \mathrm{dL}$, e valores $\geq 140 \mathrm{mg} / \mathrm{dL}$ e $<200 \mathrm{mg} / \mathrm{dL}$, após a sobrecarga com 75 g de glicose.

\section{- Variáveis sócio-demográficas}

Foram incluídos participantes de ambos os sexos. A idade, em anos completos, foi obtida mediante o cálculo: [(data da entrevista - data de nascimento)/365,25]. O nível de escolaridade foi classificado, segundo o número de anos completos de aprovação na escolaridade formal, em três categorias: 0-3 anos; 4-7 anos e 8 anos e mais. A renda mensal individual, em Reais, foi informada pelo participante utilizando-se, como referência, o mês que antecedeu a entrevista. A variável contínua, assim obtida, foi classificada segundo pontos de corte, correspondentes aos respectivos terços de renda. Os participantes que não referiram ganho mensal foram classificados como "sem renda". Considerou-se também a presença ou ausência de companheiro, no momento da entrevista, independentemente de união formal. A variável estado marital foi classificada em duas categorias: sem companheiro e com companheiro. Os participantes foram questionados sobre sua inserção no mercado de trabalho, utilizando-se, como referência, o mês que antecedeu a entrevista. Esta variável foi classificada em duas categorias: sim e não.

\section{- Fatores de risco cardiovasculares}

Os participantes foram submetidos a dosagens de colesterol total, aferidas em amostras de sangue capilar (mg/dL), após digito-punctura com lancetadores automáticos e coletadas em tiras-teste, sendo a leitura efetuada pelo método de colorimetria por reflectância, em aparelhos portáteis da marca Accutrend. Esta variável foi classificada como dicotômica, utilizando-se, como ponto de corte, valores de $200 \mathrm{mg} / \mathrm{dL}$ 16. A presença/ausência de antecedentes familiares de diabetes mellitus foi informada, tomando-se como referência os antecedentes familiares até a terceira geração de ascendentes. As respostas relacionadas à autopercepção do estado de saúde geraram uma variável classificada em duas categorias: excelente/bom e ruim/péssimo. Sobre o hábito de fumar cigarros, os participantes foram classificados em não fumantes, ex-fumantes e fumantes, sendo 
estes últimos classificados, segundo a duração do hábito, em anos completos, de acordo com os pontos de corte correspondentes aos tercis da distribuição. Medidas antropométricas da cintura e do quadril (em centímetros) foram aferidas por entrevistadores treinados e calibrados, que utilizaram fitas inelásticas da marca Seca (Seca, Hamburgo, Alemanha). Para as medidas da cintura, utilizou-se a menor curvatura situada entre o rebordo costal e a crista ilíaca. A circunferência do quadril teve como ponto de referência a maior protuberância dos glúteos (visão lateral). A altura em centímetros foi aferida por meio de estadiômetros de parede da marca Seca, sendo solicitado que os participantes retirassem os sapatos e se posicionassem com os pés unidos e contra a parede, de modo a apoiar cinco pontos do corpo: calcanhar, panturrilha, glúteos, espáduas e a cabeça que deveria estar posicionada, respeitando-se o plano de Frankfurt 17. O estadiômetro foi fixado a $2,20 \mathrm{~m}$ verticais, em relação ao piso, e calibrado com esquadro técnico de 60 graus que foi também utilizado para encontrar o ângulo reto entre o piso e a parede onde o estadiômetro deveria ser fixado. De cada participante foram obtidas duas aferições para as medidas antropométricas, utilizando-se, respectivamente, a média destas duas medidas. Para o propósito deste estudo, utilizaram-se a circunferência da cintura (pontos de corte específicos, segundo o sexo) 18 e as razões cintura/quadril e cintura/ altura, classificadas segundo os pontos de corte correspondentes aos tercis da distribuição.

\section{- Fatores comportamentais}

A atividade física foi aferida mediante a aplicação do International Physical Activity Questionnaire (IPAQ versão curta) ${ }^{19}$, sendo os participantes classificados como: muito ativos; moderadamente ativos e insuficientemente ativos. Além da atividade física, os participantes foram convidados a responder questões relacionadas ao uso de medicamentos (nos 15 dias que antecederam a entrevista), à procura por serviços ambulatoriais de saúde (nos últimos seis meses) e à ocorrência de internações hospitalares (nos últimos dois anos), sendo as duas últimas classificadas de forma dicotômica como sim/não. O número de medicamentos foi classificado em quatro categorias: não tomou; tomou 1-2; tomou 3-4 e tomou 5 ou mais.

\section{Processamento dos dados}

A coleta dos dados foi conduzida mediante a aplicação de entrevistas estruturadas, aplicadas nos domicílios dos elegíveis, por uma equipe de entrevistadores, previamente treinada. Antes da digitação definitiva (digitação com dupla entrada de dados), o controle de qualidade das informações foi avaliado, mediante a replicação de 12,5\% das entrevistas, obtendo-se a estatística kappa 20 para a avaliação da reprodutibilidade das informações. O coeficiente kappa, para todas as questões incluídas na replicação foi superior a 0,80.

\section{Análise estatística dos dados}

Prevalências brutas e respectivos IC95\% foram calculados para o diabetes mellitus (variáveldesfecho) e para as classes tolerância diminuída à glicose e glicemia de jejum alterada. Posteriormente, a prevalência de diabetes mellitus foi estimada por pontos e por IC95\%, em estratos específicos dos fatores sócio-demográficos, fatores de risco cardiovasculares e fatores comportamentais. Na fase descritiva do estudo, associações globais entre os fatores supramencionados e o diabetes mellitus foram testadas utilizandose a estatística F, com correção para efeito de desenho, adotando-se nível de significância de $5 \%$. Na fase analítica, utilizou-se a regressão de Poisson ${ }^{21}$ para obtenção das razões de prevalências (RP) que foram estimadas por pontos e por IC95\%. Inicialmente, foram efetuadas análises univariadas, selecionando-se para os modelos subseqüentes, as variáveis com valores $\mathrm{p} \leq 0,25$ para a estatística de Wald. A seguir, as razões de prevalências foram estimadas em modelos parciais de ajustamento, contendo, respectivamente, fatores sócio-demográficos, fatores de risco cardiovasculares e fatores comportamentais. Em etapa subseqüente, iniciou-se a construção do modelo final, incluindo-se as variáveis que, dentro de cada um dos três grupos de fatores, acima descritos, apresentaram valores de $\mathrm{p}<0,05$ para a estatística de Wald. O modelo final foi composto pelas variáveis que, no decorrer desta etapa, mantiveram significância estatística $(\mathrm{p}<0,05)$.

As estimativas foram calculadas, levando-se em consideração o efeito de desenho 14, utilizando-se módulo específico (survey) do programa Stata, versão 8.2 (Stata Corp., College Station, Estados Unidos). A utilização dos comandos svy do módulo survey do Stata permite o cálculo de variâncias em amostras complexas, visto que o sorteio em diferentes estágios retira a eqüiprobabilidade de sorteio que ocorre ao se considerar o processo de amostragem casual simples.

\section{Considerações éticas}

O Projeto OBEDIARP foi aprovado pelo Comitê de Ética em Pesquisa da Escola de Enfermagem de Ribeirão Preto, Universidade de São Paulo e 
protocolado sob o $\mathrm{n}^{\mathrm{o}}$. $0528 / 2005$. Todos os participantes assinaram o Termo de Consentimento Livre e Esclarecido, conforme recomendações da Resolução $n^{o}$. 196/96 do Conselho Nacional de Saúde.

\section{Resultados}

No Projeto OBEDIARP, a amostra de participantes foi representativa da população urbana, residente em Ribeirão Preto, apresentando distribuição percentual segundo a idade semelhante ao Censo Demográfico 2000, em ambos os sexos.

Na Tabela 1, apresentam-se as prevalências de diabetes mellitus e das demais classes de intolerância à glicose (tolerância diminuída à glicose e glicemia de jejum alterada). Observa-se elevada prevalência de diabetes mellitus na população estudada (15,02\%; IC95\%: 12,68-17,36). As prevalências para a tolerância diminuída à glicose e para a glicemia de jejum alterada foram, respectivamente, 5,53\% (IC95\%: 3,93-7,13) e 5,46\% (IC95\%: 3,87-7,06). O efeito de desenho (deff) foi equivalente a 1,0619.

Entre os participantes classificados como diabéticos (nw = 327,8), cerca de $15 \%$ desconheciam a condição antes da pesquisa.

As Tabelas 2, 3 e 4 exibem, respectivamente, as prevalências de diabetes mellitus, em estratos específicos de fatores sócio-demográficos, fatores de risco cardiovasculares e fatores comportamentais.

Em relação aos fatores sócio-demográficos (Tabela 2), observa-se que as variáveis faixa etária, escolaridade, renda e condição de trabalho apresentaram associação com o diabetes mellitus ( $\mathrm{p}$ para a estatística $\mathrm{F}<0,05$ ). A prevalência de diabetes mellitus apresentou relação direta com a idade e relação inversa com a renda, a escolaridade e a condição de trabalho, observando-se tendência linear ( $p$ de tendência $<0,05$ ) nas categorias das variáveis: faixa etária, escolaridade e renda.

Em relação aos fatores de risco cardiovasculares (Tabela 3), observa-se que as variáveis: antecedentes familiares de diabetes mellitus; autopercepção do estado de saúde; duração do hábito de fumar; circunferência da cintura e as razões cintura/quadril e cintura/altura apresentaram associação com o diabetes mellitus (valor de p para a estatística $\mathrm{F}<0,05$ ). As prevalências de diabetes mellitus apresentaram gradiente linear ( $p$ de tendência $<0,05$ ) nos respectivos tercis das razões cintura/quadril e cintura/altura.

Na Tabela 4 observa-se que o número de medicamentos consumidos nos últimos 15 dias e a procura por serviços de saúde, nos últimos seis meses, apresentaram associação com o diabetes mellitus, destacando-se o gradiente linear da prevalência de diabetes mellitus, nas categorias da variável número de medicamentos ( $p$ de tendência <0,05).

Na Tabela 5 apresenta-se o modelo final (modelo multivariado) que foi constituído pelo seguinte elenco de variáveis, independentemente associadas ao diabetes mellitus: faixa etária; antecedentes familiares de diabetes mellitus; razões cintura/quadril e cintura/altura; número de medicamentos consumidos nos últimos 15 dias

Tabela 1

Prevalência de classes de tolerância à glicose *, e intervalos de 95\% de confiança (IC95\%). Ribeirão Preto, São Paulo, Brasil, 2006, Projeto OBEDIARP.

\begin{tabular}{lccc}
\hline & nw ** & $\%$ & IC95\% \\
\hline Normal & $1.614,0$ & 73,96 & $71,03-76,90$ \\
Diabetes mellitus & 327,8 & 15,02 & $12,68-17,36$ \\
Tolerância à glicose diminuída & 120,8 & 5,53 & $3,93-7,13$ \\
Glicemia de jejum alterada & 119,3 & 5,46 & $3,87-7,06$ \\
Total & $2.182,0$ & 100,00 & - \\
\hline
\end{tabular}

* Pontos de corte recomendados pela Organização Mundial da Saúde 15;

** Amostra ponderada: 15 participantes foram excluídos por se recusarem a realizar as dosagens de glicemias.

e procura por serviços de saúde. Em todas as variáveis com mais de duas categorias que permaneceram no modelo final, observa-se tendência linear para as respectivas razões de prevalência ( $\mathrm{p}<0,05$ ), antes e após o ajustamento para potenciais fatores de confusão.

\section{Discussão}

Os resultados permitiram identificar elevada prevalência de diabetes mellitus na população do estudo. Menor proporção de diabéticos que desconheciam a condição antes da pesquisa (15\%), em relação ao estudo de Malerbi \& Franco 6 (50\%), pode ter sido decorrente, entre outros, dos seguintes fatores: (a) maior acesso e disponibilidade de serviços de saúde, em relação à década de 80; (b) implantação e manutenção de programas de identificação de casos e (c) elevado nível de escolaridade da população, o que certamente contribuiu para o diagnóstico precoce.

No presente estudo, além da composição de amostra representativa de adultos residentes no município, destaca-se o rigor metodológico com 
Tabela 2

Prevalência de diabetes mellitus *, com respectivos intervalos de 95\% de confiança (IC95\%), segundo fatores sóciodemográficos. Ribeirão Preto, São Paulo, Brasil, 2006, Projeto OBEDIARP.

\begin{tabular}{|c|c|c|c|c|}
\hline \multirow[t]{2}{*}{ Fatores sócio-demográficos } & \multicolumn{2}{|c|}{ Normal $(n w=1.614,0) * \star$} & \multicolumn{2}{|c|}{ Diabetes mellitus $(\mathrm{nw}=327,8)$ ** } \\
\hline & $\%$ & IC95\% & $\%$ & IC95\% \\
\hline \multicolumn{5}{|l|}{ Sexo } \\
\hline Masculino & 83,40 & $78,18-87,57$ & 16,60 & $12,43-21,82$ \\
\hline Feminino & 83,00 & $79,67-85,88$ & 17,00 & $14,12-20,33$ \\
\hline \multicolumn{5}{|l|}{ Faixa etária (anos) $\star \star \star, \#$} \\
\hline $30-39$ & 93,87 & $89,66-96,44$ & 6,13 & $3,56-10,34$ \\
\hline $40-49$ & 90,33 & $86,02-93,41$ & 9,67 & 6,59-13,98 \\
\hline $50-59$ & 75,17 & $68,55-80,78$ & 24,83 & $19,22-31,45$ \\
\hline 60 e mais & 61,13 & $52,19-69,38$ & 38,87 & $30,62-47,81$ \\
\hline \multicolumn{5}{|l|}{ Escolaridade (anos) $* \star \star, \#$} \\
\hline $0-3$ & 70,30 & $61,70-77,67$ & 29,70 & $22,33-38,30$ \\
\hline $4-7$ & 80,84 & $75,12-85,49$ & 19,16 & $14,51-24,88$ \\
\hline 8 e mais & 87,95 & $84,24-90,88$ & 12,05 & $9,12-15,76$ \\
\hline \multicolumn{5}{|l|}{ Renda individual $(\mathrm{R} \$) * \star \star, \#$} \\
\hline Sem renda & 75,75 & $70,25-80,51$ & 24,25 & $19,49-29,75$ \\
\hline 1ㅇ terço $(\geq 60,00 \leq 520,00)$ & 83,55 & $77,26-88,36$ & 16,45 & $11,64-22,74$ \\
\hline 2o terço $(>520,00 \leq 1.100,00)$ & 87,93 & $82,16-92,02$ & 12,07 & $7,98-17,84$ \\
\hline 3ㅇ terço $(>1.100,00)$ & 90,73 & $85,56-94,18$ & 9,27 & $5,82-14,44$ \\
\hline \multicolumn{5}{|l|}{ Estado marital } \\
\hline Sem companheiro & 82,15 & $76,32-86,79$ & 17,85 & $13,21-23,68$ \\
\hline Com companheiro & 83,52 & $80,17-86,41$ & 16,48 & $13,59-19,83$ \\
\hline \multicolumn{5}{|l|}{ Condição trabalho *** } \\
\hline Não & 75,75 & $70,25-80,51$ & 24,25 & $19,49-29,75$ \\
\hline Sim & 87,43 & $84,33-90,00$ & 12,57 & $10,00-15,67$ \\
\hline
\end{tabular}

* Pontos de corte recomendados pela Organização Mundial da Saúde 15;

** Amostra ponderada;

*** Valor de $\mathrm{p}$ para a estatística $\mathrm{F}<0,05$

\# Valor de p para o teste de tendência linear $<0,05$.

que foram conduzidas todas as suas etapas: processo de amostragem; treinamento e calibração dos entrevistadores para a coleta de dados 22 ; consideração de história prévia de diabetes mellitus, combinada com a aferição de glicemias de jejum e pós-sobrecarga para a classificação da intolerância à glicose; replicação de 12,5\% de entrevistas para avaliação do controle de qualidade das informações e elevada taxa de resposta (78\%), que, em conjunto, reforçam a validade interna do estudo e, por conseguinte, a convicção de que os resultados encontrados não foram decorrentes de vícios sistemáticos.

No Brasil, poucos estudos de base populacional têm sido desenvolvidos para a detecção da prevalência de diabetes mellitus, merecendo destaque os resultados publicados, em 1992, por Malerbi \& Franco ${ }^{6}$, sobre a prevalência de diabetes mellitus em nove capitais brasileiras. A partir da década de 80 , nenhum outro estudo com re- presentatividade nacional foi desenvolvido com o propósito de investigar a prevalência de diabetes mellitus.

No período entre 2001-2003, graças à iniciativa do Ministério da Saúde, em parceria com a Organização Pan-Americana da Saúde (OPAS), 22,1 milhões de brasileiros, com 40 anos e mais, participaram da Campanha Nacional de Detecção do Diabetes Mellitus (CNDDM) 23 e, entre eles, $16 \%$ foram positivos na fase de rastreamento. Entre os que apresentaram alterações da glicemia de jejum de leve a moderada (normal elevada + resultado duvidoso + resultado alterado) e que compareceram às unidades de referência para a segunda etapa da campanha, $14,02 \%$ tiveram o diagnóstico de diabetes mellitus confirmado. Diferenças relacionadas às características dos municípios que participaram da campanha $(90 \%$ dos municípios possuíam menos de 50 mil habitantes), bem como diferenças quanto ao método 
Tabela 3

Prevalência de diabetes mellitus *, com respectivos intervalos de $95 \%$ de confiança (IC95\%), segundo fatores de risco cardiovasculares. Ribeirão Preto, São Paulo, Brasil, 2006, Projeto OBEDIARP.

\begin{tabular}{|c|c|c|c|c|}
\hline \multirow[t]{2}{*}{ Fatores de risco } & \multicolumn{2}{|c|}{ Normal $(n w=1.614,0) * \star$} & \multicolumn{2}{|c|}{ Diabetes mellitus ( $\mathrm{nw}=327,8)$ ** } \\
\hline & $\%$ & IC95\% & $\%$ & IC95\% \\
\hline \multicolumn{5}{|l|}{ Colesterol total (mg/dL) } \\
\hline$<200$ & 84,56 & $81,14-87,45$ & 15,44 & $12,55-18,86$ \\
\hline$\geq 200$ & 78,70 & $71,42-84,52$ & 21,30 & $15,48-28,58$ \\
\hline \multicolumn{5}{|l|}{ Antecedentes familiares } \\
\hline \multicolumn{5}{|l|}{ 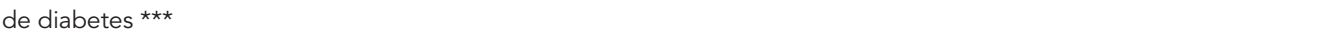 } \\
\hline Sem antecedentes & 87,88 & $84,17-90,82$ & 12,12 & $9,18-15,83$ \\
\hline Com antecedentes & 78,75 & $74,25-82,65$ & 21,25 & $17,35-25,75$ \\
\hline \multicolumn{5}{|l|}{ 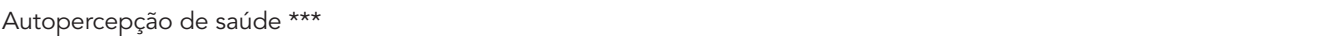 } \\
\hline Excelente/Bom & 87,17 & $83,78-89,94$ & 12,83 & $10,06-16,22$ \\
\hline Ruim/Péssimo & 74,98 & $70,34-79,11$ & 25,02 & $20,89-29,66$ \\
\hline \multicolumn{5}{|l|}{ Hábito de fumar } \\
\hline Não fumante & 84,76 & $81,32-87,66$ & 15,24 & $12,34-18,68$ \\
\hline Ex-fumante & 77,11 & $70,88-82,34$ & 22,89 & $17,66-29,12$ \\
\hline Fumante & 85,75 & $79,76-90,19$ & 14,25 & $9,81-20,24$ \\
\hline \multicolumn{5}{|l|}{ Tempo de fumo (anos) $\star \star \star ~$} \\
\hline Não fumante & 84,86 & $81,45-87,74$ & 15,14 & $12,26-18,55$ \\
\hline 1 o terço $(>0 \leq 13,63)$ & 84,26 & $76,76-89,67$ & 15,74 & $10,33-23,24$ \\
\hline 2o terço $(>13,63 \leq 25)$ & 86,28 & $79,05-91,28$ & 13,72 & $8,72-20,95$ \\
\hline 3o terço (> 25) & 72,83 & $64,24-80,01$ & 27,17 & $19,99-35,76$ \\
\hline \multicolumn{5}{|l|}{ Circunferência da cintura $* \star \star, \#$} \\
\hline Normal & 90,96 & $87,02-93,79$ & 9,04 & $6,21-12,98$ \\
\hline Alterada & 76,42 & $72,22-80,15$ & 23,58 & $19,85-27,78$ \\
\hline \multicolumn{5}{|l|}{ Razão cintura/quadril ***,\#\# } \\
\hline 1 o terço $(\leq 0,8077)$ & 92,24 & $88,25-94,96$ & 7,76 & $5,04-11,75$ \\
\hline 2o terço $(>0,8077 \leq 0,8775)$ & 84,56 & $80,26-88,07$ & 15,44 & $11,93-19,74$ \\
\hline 3o terço $(>0,8775)$ & 71,27 & $65,62-76,32$ & 28,73 & $23,68-34,38$ \\
\hline \multicolumn{5}{|l|}{ Razão cintura/altura ***,\#\# } \\
\hline 1ㅇ terço $(\leq 0,5014)$ & 93,96 & $90,05-96,40$ & 6,04 & $3,60-9,95$ \\
\hline 2o $\operatorname{terço~}(>0,5014 \leq 0,5661)$ & 86,77 & $81,62-90,64$ & 13,23 & $9,36-18,38$ \\
\hline 3o $\operatorname{terço~}(>0,5661)$ & 66,01 & $60,46-71,15$ & 33,99 & $28,85-39,54$ \\
\hline
\end{tabular}

* Pontos de corte recomendados pela Organização Mundial da Saúde 15 .

** Amostra ponderada;

*** Valor de p para a estatística $F<0,05$;

\# Pontos de corte: masculino $\geq 94 \mathrm{~cm}$ e feminino $\geq 80 \mathrm{~cm}$, de acordo com Albert et al. 18;

\#\# Valor de p para o teste de tendência linear $<0,05$

e critérios diagnósticos adotados (alterações da glicemia de jejum, segundo pontos de corte adotados na CNDDM) impedem comparações diretas com os resultados do Projeto OBEDIARP. Entretanto, as limitações impostas pela utilização de diferentes metodologias para a investigação do diabetes mellitus (campanhas de rastreamento versus estudos de prevalência) não impedem a formulação da hipótese de que o contingente de casos confirmados pela CNDDM poderia até ser mais elevado, se as perdas de participantes, na etapa de confirmação diagnóstica, não tivessem sido tão elevadas $(48,9 \%)$ e, se testes orais de tolerância à glicose, além de glicemias plasmáticas de jejum, fossem aplicados em sub-amostras de participantes com glicemia de jejum normal. Há que se considerar, por outro lado, que a CNDDM desencadeou a Portaria Conjunta do Ministério da Saúde n. ${ }^{\circ}$ 112, de 19 de junho de 2002 que dispõe sobre "a necessidade de monitoramento, em nível nacional, do Programa de Assistência Farmacêutica à Hipertensão e ao Diabetes Mellitus, por intermédio do Sistema de Cadastramento $e$ Acompanhamento de Hipertensos e Diabéticos 
Prevalência de diabetes mellitus *, com respectivos intervalos de 95\% de confiança (IC95\%), segundo fatores comportamentais e utilização de serviços de saúde. Ribeirão Preto, São Paulo, Brasil, 2006, Projeto OBEDIARP.

\begin{tabular}{|c|c|c|c|c|}
\hline \multirow{3}{*}{$\begin{array}{l}\text { Fatores comportamentais } \\
\text { e utilização de serviços } \\
\text { de saúde }\end{array}$} & \multicolumn{2}{|c|}{ Normal $(n w=1.614,0) * \star$} & \multicolumn{2}{|c|}{ Diabetes mellitus ( $\mathrm{nw}=327,8)$ ** } \\
\hline & $\%$ & IC95\% & $\%$ & IC95\% \\
\hline & & & & \\
\hline \multicolumn{5}{|l|}{ Atividade física } \\
\hline Muito ativos & 88,38 & $79,14-93,84$ & 11,62 & $6,16-20,86$ \\
\hline Moderadamente ativos & 82,65 & $77,50-86,82$ & 17,35 & $13,18-22,50$ \\
\hline Insuficientemente ativos & 82,58 & $79,10-85,58$ & 17,42 & $14,42-20,90$ \\
\hline \multicolumn{5}{|c|}{ Número de medicamentos ***,\# } \\
\hline Não tomou & 91,42 & $86,50-94,66$ & 8,58 & $5,34-13,50$ \\
\hline Tomou 1-2 & 89,82 & $86,32-92,50$ & 10,18 & $7,50-13,68$ \\
\hline Tomou 3-4 & 79,49 & $72,45-85,10$ & 20,51 & $14,90-27,55$ \\
\hline Tomou 5 ou mais & 46,31 & $35,93-57,03$ & 53,69 & $42,97-64,07$ \\
\hline \multicolumn{5}{|l|}{ 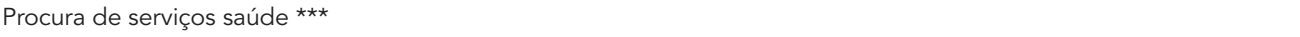 } \\
\hline Não & 93,82 & $89,62-96,39$ & 6,18 & $3,61-10,38$ \\
\hline Sim & 79,73 & $76,35-82,73$ & 20,27 & $17,27-23,65$ \\
\hline \multicolumn{5}{|l|}{ Internação nos últimos 2 anos } \\
\hline Não & 83,95 & $80,89-86,61$ & 16,05 & $13,39-19,11$ \\
\hline Sim & 79,78 & $72,62-85,45$ & 20,22 & $14,55-23,38$ \\
\hline
\end{tabular}

* Pontos de corte recomendados pela Organização Mundial da Saúde 15;

** Amostra ponderada;

$\star * *$ Valor de $p$ descritivo para a estatística $F<0,05$;

\# Valor de p para o teste de tendência linear $<0,05$.

(HiperDia)" 24, considerada a primeira base de dados com cobertura nacional para a notificação de casos de doenças crônicas.

No Município de Ribeirão Preto, em estudo de base populacional conduzido entre 1996 e 1997, em amostra de 1.473 participantes com idade entre 30 e 69 anos, Torquato et al. 25 detectaram que as prevalências de diabetes mellitus e de tolerância diminuída à glicose foram, respectivamente, $12,1 \%$ e $7,7 \%$. Embora os resultados tenham sido apresentados apenas sob a forma descritiva, os autores detectaram que a prevalência de diabetes mellitus aumentou com a idade, foi mais elevada em participantes obesos e com antecedentes familiares de diabetes mellitus e diminuiu com a escolaridade, resultados estes, semelhantes aos do presente estudo. No Projeto OBEDIARP, prevalência de diabetes mellitus com magnitude mais elevada que a encontrada por Torquato et al. 25, em 1997, é resultante, certamente, do envelhecimento populacional relativo, já observado no Município de Ribeirão Preto, e que é responsável, como esperado, pelo acúmulo desta doença na população.

Resultados de um estudo transversal conduzido entre 1999 e 2000 por Schaan et al. 26, em amostra representativa da população do Estado do Rio Grande do Sul indicaram prevalências de $12,4 \%$ e de $7,4 \%$ para o diabetes mellitus e para a glicemia de jejum alterada, respectivamente. Neste estudo, os participantes com algum grau de alteração da homeostase glicêmica apresentaram maior prevalência de obesidade, hipertensão arterial e hipercolesterolemia, em relação aos classificados como normais.

Dentre os poucos estudos analíticos existentes no Brasil, cuja proposta foi a detecção da prevalência de diabetes mellitus e de fatores associados, inclui-se o Bambuí Health and Aging Study, estudo prospectivo de base populacional, iniciado em 1997 na cidade de Bambuí, Minas Gerais. Utilizando dados referentes à primeira visita da coorte, Passos et al. 27 detectaram prevalência de diabetes mellitus, em adultos, de 2,33\% (18 a 59 anos) e de 14,59\%, em idosos (60 anos e mais) sendo que as prevalências de glicemia de jejum alterada corresponderam a 5,64\% (adultos) e $13,32 \%$ (idosos). Na fase analítica, os autores identificaram os seguintes fatores associados ao diabetes mellitus: razão cintura/quadril (OR = 4,99; IC95\%: 1,47-17,00) e colesterol plasmático $\geq 240 \mathrm{mg} / \mathrm{dL}$ (OR = 3,22; IC95\%: 1,14-9,12), que permaneceram nos modelos finais para os adultos (18 a 59 anos). Entre os idosos (60 anos 
Razões de prevalência (RP) brutas e ajustadas, com respectivos intervalos de 95\% de confiança (IC $95 \%$ ) e valores de p para os testes de tendência linear: modelo final. Ribeirão Preto, São Paulo, Brasil, 2006, Projeto OBEDIARP.

\begin{tabular}{|c|c|c|c|c|c|c|}
\hline Variáveis & RP bruta & IC95\% & Valor de $p$ & RP ajustada & IC95\% & Valor de $\mathrm{p}$ \\
\hline \multicolumn{7}{|l|}{ Faixa etária (anos) } \\
\hline $30-39$ & 1,00 & & & 1,00 & & \\
\hline $40-49$ & 1,57 & $0,79-3,13$ & & 1,24 & $0,62-2,47$ & \\
\hline $50-59$ & 4,05 & $2,28-7,19$ & 0,000 & 2,22 & $1,14-4,32$ & 0,002 \\
\hline 60 e mais & 6,34 & $3,43-11,72$ & & 2,62 & $1,31-5,24$ & \\
\hline \multicolumn{7}{|l|}{ Antecedente familiar de diabetes } \\
\hline Sem antecedentes & 1,00 & & & 1,00 & & \\
\hline Com antecedentes & 1,75 & $1,24-2,46$ & - & 1,74 & $1,28-2,37$ & - \\
\hline \multicolumn{7}{|l|}{ Razão cintura/quadril } \\
\hline 1ㅇ terço $(\leq 0,8077)$ & 1,00 & & & 1,00 & & \\
\hline 2o terço $(>0,8077 \leq 0,8775)$ & 1,99 & $1,27-3,11$ & 0,000 & 1,09 & $0,69-1,73$ & 0,001 \\
\hline 3o terço $(>0,8775)$ & 3,70 & $2,36-5,81$ & & 1,70 & $1,04-2,76$ & \\
\hline \multicolumn{7}{|l|}{ Razão cintura/altura } \\
\hline 1으 terço $(\leq 0,5014)$ & 1,00 & & & 1,00 & & \\
\hline 2o $\operatorname{terço~}(>0,5014 \leq 0,5661)$ & 2,19 & $1,22-3,93$ & 0,000 & 1,30 & $0,69-2,44$ & 0,003 \\
\hline 3o terço $(>0,5661)$ & 5,62 & $3,31-9,55$ & & 2,39 & $1,30-4,40$ & \\
\hline \multicolumn{7}{|l|}{ Número de medicamentos } \\
\hline Não tomou & 1,00 & & & 1,00 & & \\
\hline Tomou 1-2 & 1,18 & $0,71-1,98$ & & 1,15 & $0,69-1,90$ & \\
\hline Tomou 3-4 & 2,39 & $1,32-4,29$ & 0,000 & 1,43 & $0,83-2,45$ & 0,000 \\
\hline Tomou 5 ou mais & 6,25 & $3,66-10,68$ & & 2,61 & $1,57-4,34$ & \\
\hline \multicolumn{7}{|l|}{ Procura de serviços saúde } \\
\hline Não & 1,00 & & & 1,00 & & \\
\hline Sim & 3,28 & $1,89-5,68$ & - & 2,13 & $1,21-3,73$ & - \\
\hline
\end{tabular}

e mais), permaneceram nos modelos finais as seguintes variáveis: antecedentes familiares de diabetes $(\mathrm{OR}=1,92$; IC95\%: 1,36-2,73); razão cintura/quadril (OR = 1,79; IC95\%: 1,25-2,56) e triglicérides plasmáticos entre 200-499mg/dL (OR = 1,71; IC95\%: 1,13-2,59).

Martinez \& Latorre 28, em estudo transversal conduzido em 1997, e no qual foram incluídos 3.777 trabalhadores dos ramos da indústria metalúrgica e siderúrgica, nos estados de São Paulo e Rio de Janeiro, detectaram prevalência de diabetes mellitus de $11,5 \%$. Utilizando modelos múltiplos hierarquizados, os autores identificaram o excesso de peso e as dislipidemias (variáveis de nível proximal, no modelo) como fatores associados ao diabetes mellitus ( $p<0,001$ ). A comparabilidade destes resultados com os do presente estudo é limitada, visto que, além de não considerarem as glicemias pós-sobrecarga a amostra do estudo foi constituída por voluntários, embora representantes, segundo os autores, de $50 \%$ da classe trabalhadora, neste seguimento da indústria.
Além da escassez de estudos desenvolvidos com amostras representativas da população tipicamente brasileira para estimativas da prevalência de diabetes mellitus e fatores associados, outra limitação no que se refere à comparabilidade dos resultados está relacionada aos métodos adotados para o diagnóstico de diabetes mellitus. Nos estudos desenvolvidos no Brasil por Malerbi \& Franco 6, Torquato et al. 25, Schaan et al. 26 e Passos et al. 27, os autores utilizaram os critérios propostos pela OMS para o estabelecimento de pontos de corte referentes à glicemia de jejum e à glicemia pós-sobrecarga (sangue capilar ou plasma venoso), que são de fundamental importância para a confirmação diagnóstica de diabetes mellitus e das classes de intolerância: tolerância diminuída à glicose e glicemia de jejum alterada, além de história prévia de diabetes mellitus.

Por outro lado, em estudo de base populacional para estimar a prevalência de diabetes mellitus em Pelotas, Rio Grande do Sul 29, os critérios adotados foram história prévia de diabetes 
mellitus (confirmada pelo médico) em amostra de 1.968 participantes e dosagens de glicemias de jejum em sub-amostra de 367 participantes, utilizando os pontos de corte 126 e $140 \mathrm{mg} / \mathrm{dL}$ que foram combinados com história prévia de diabetes mellitus. As prevalências, ao utilizarem a história prévia de diabetes mellitus para os 1.968 e para a sub-amostra de 367 participantes foram, respectivamente, 5,6\% e 7,1\%. Ao utilizarem os pontos de corte supracitados para as glicemias de jejum, combinados com história prévia de diabetes mellitus, as respectivas prevalências foram $10,3 \%$ e $9 \%$. Tendo em vista a não disponibilidade das glicemias pós-sobrecarga, os autores não conseguiram incluir nas estimativas de prevalência, os diabéticos "não clinicamente manifestos" que podem apresentar glicemias de jejum normais (alteração apenas da fase I de secreção de insulina-secreção rápida) 30 , tornando-se imprescindível, para a sua captura, os valores glicêmicos de pós-sobrecarga. Depreende-se, portanto, que a aferição isolada das glicemias de jejum pode subestimar a verdadeira prevalência de diabetes mellitus na população.

No Projeto OBEDIARP, as razões cintura/ quadril e cintura/altura, indicadores de obesidade regional, permaneceram nos modelos finais, sendo que, para ambas, foi observada a presença de efeito dose-resposta, em relação ao diabetes mellitus $(p<0,05)$. A opção por utilizar estas medidas, ao invés do índice de massa corporal (IMC), parece pertinente, visto que Lee et al. 31 em uma meta-análise, na qual, modelos de efeitos aleatórios foram aplicados a dez estudos, detectaram que a razão cintura/altura apresentou áreas sob as curvas ROC de maior magnitude na detecção de diabetes mellitus, hipertensão arterial e dislipidemias que o IMC, sendo 0,73 (IC95\%: 0,70-0,75) para o sexo masculino e 0,76 (IC95\%: 0,70-0,81) para o sexo feminino. Kagawa et al. ${ }^{32}$, em estudo de validação conduzido na Austrália, ao utilizarem a razão cintura/altura, sendo a circunferência da cintura aferida como a menor curvatura entre o rebordo costal e a crista ilíaca, identificaram que este indicador apresentou elevada especificidade para a detecção de obesidade regional (porcentagem de gordura no tronco), sendo equivalente a $92,3 \%$ para o sexo masculino e $100 \%$ para o sexo feminino. Estes resultados, em conjunto, conferem consistência àqueles obtidos no Projeto OBEDIARP onde foi utilizada a mesma técnica para a aferição da circunferência da cintura, tendo a razão cintura/ altura permanecido no elenco de variáveis associadas ao diabetes mellitus (modelo final).

$\mathrm{O}$ avanço do conhecimento em biologia molecular permitiu a identificação das adipocinas, que atuam como elos moleculares entre obesidade e doença. O aumento da adiposidade visceral associa-se com o aumento da prevalência de diabetes mellitus e da doença cardíaca coronariana, e isto ocorre mediante a expressão ou secreção alterada, no tecido adiposo branco, de um conjunto de fatores relacionados à resistência insulínica e a fatores pró-inflamatórios e próaterogênicos 33 . Embora níveis séricos de leptina ou adiponectina não tenham sido dosados neste estudo e, portanto, não façam parte do elenco de variáveis independentes, a associação detectada entre obesidade regional e diabetes mellitus, ao que parece, pode representar, fenotipicamente, a expressão da ativação destas vias moleculares.

No presente estudo, além das razões cintura/ quadril e cintura/altura, as variáveis faixa etária, antecedentes familiares de diabetes mellitus, número de medicamentos e procura por serviços de saúde estiveram associadas com o diabetes mellitus, detectando-se tendência linear para as razões de prevalências das variáveis faixa etária razões cintura/quadril e cintura/altura e número de medicamentos.

Estudos conduzidos em diferentes países têm evidenciado a consistência de associações entre medidas de obesidade central e o diabetes mellitus 34,35,36,37,38,39, destacando-se, entre estas, a razão cintura/altura que, além de permanecer em modelos finais apresentou maior acurácia na predição do desfecho (áreas sob curvas ROC), em relação aos demais indicadores de obesidade central ou de obesidade global 31,32,38,39.

No Projeto OBEDIARP, a presença de antecedentes familiares de diabetes mellitus esteve fortemente associada ao diabetes mellitus no modelo final (RP = 1,74; IC95\%: 1,28-2,37), corroborando os achados de Bener et al. 34 e Hadaegh et al. 35 .

$\mathrm{Na}$ fase descritiva do presente estudo as variáveis faixa etária, escolaridade e renda apresentaram relação linear com o diabetes mellitus. Entretanto, apenas a variável faixa etária, entre as sócio-demográficas, permaneceu no modelo final. Resultados do Alameda County Study, publicados em 2005 por Maty et al. 40,41 indicaram associação entre o nível educacional e o diabetes mellitus, após o ajustamento para as variáveis renda e ocupação. Esta associação, contudo, não foi confirmada, após o ajustamento para inatividade física, hábito de fumar, consumo de álcool, composição corporal, hipertensão arterial, depressão e acesso a serviços de saúde.

No Projeto OBEDIARP, as variáveis: hábito de fumar e sua duração, padrão de atividade física e hipercolesterolemia não apresentaram associação com o diabetes mellitus. A impossibilidade de aferir frações de colesterol (HDL, 
LDL e VLDL), bem como níveis de triglicérides em equipamentos portáteis, limitou a investigação detalhada do efeito das dislipidemias sobre o diabetes mellitus. Embora a classificação da prática de atividade física bem como do tempo sentado tenham sido pautadas ipsis literis nas recomendações contidas no protocolo do IPAQ, estas variáveis não detiveram bom poder discriminatório, talvez, em decorrência do fato de não serem mutuamente exclusivas.

A associação entre o hábito de fumar e o diabetes mellitus parece não estar ainda totalmente esclarecida, detectando-se resultados conflitantes na literatura, como aqueles publicados por Bener et al. 34 e Hadaegh et al. 35 .

Algumas limitações dos estudos transversais referem-se ao viés de causalidade reversa 13 , de- corrente de aferições simultâneas dos fatores de risco/proteção e dos desfechos, limitando inferências sobre a direcionalidade de algumas associações. O efeito independente das variáveis número de medicamentos e procura por serviços de saúde sobre o diabetes mellitus parece ser decorrente deste viés.

No que pesem as limitações dos estudos transversais, os resultados do Projeto OBEDIARP confirmaram a relevância do diabetes mellitus, como um problema de saúde pública, e permitiram identificar um elenco de fatores associados, entre os quais, alguns passíveis de intervenção, constituindo-se em importante contribuição para a construção racional de políticas públicas de promoção e prevenção em saúde no Município de Ribeirão Preto.

\section{Resumo}

Para investigar a prevalência de diabetes mellitus e fatores associados, conduziu-se estudo epidemiológico transversal em amostra de adultos com 30 anos e mais, residentes em Ribeirão Preto, São Paulo, Brasil. O processo de amostragem foi desenvolvido em três estágios e pesos amostrais foram calculados, obtendo-se amostra ponderada de 2.197 participantes. Para o diagnóstico de diabetes mellitus considerou-se história prévia e pontos de corte recomendados pela Organização Mundial da Saúde (OMS) para glicemias de jejum e pós-sobrecarga. Para identificação de fatores associados, razões de prevalências brutas e ajustadas foram estimadas por pontos e por intervalos, utilizando-se regressão de Poisson. A prevalência de diabetes mellitus foi 15,02\%. Após o ajustamento para potenciais fatores de confusão, as seguintes variáveis permaneceram no modelo final: faixa etária; antecedentes familiares de diabetes mellitus; razão cintura/quadril; razão cinturalaltura; uso de medicamentos e procura por serviços de saúde. Os resultados apontaram elevada prevalência de diabetes mellitus e permitiram a identificação de fatores associados, passíveis de intervenção.

\section{Colaboradores}

S. A. Moraes elaborou a proposta do estudo, participou de todas as etapas das análises de dados, interpretação e discussão dos resultados, foi responsável por toda a revisão bibliográfica e pela elaboração de todas as versões do manuscrito. I. C. M. Freitas participou da elaboração da proposta do estudo, colaborou no gerenciamento dos dados e em todas as etapas da análise dos dados, na discussão dos resultados e na revisão de todas as versões do manuscrito. S. G. A. Gimeno e L. Mondini colaboraram na elaboração da proposta do estudo, na discussão dos resultados e revisão da versão final do manuscrito.

\section{Agradecimentos}

O Projeto OBEDIARP foi financiado pelo Conselho Nacional de Desenvolvimento Científico e Tecnológico (CNPq), Edital MCT-Saúde no. 30 (processo no. 505622/2004-1). I. C. M. Freitas recebeu bolsa de doutorado CNPq (processo no. 141102/2007-1). 


\section{Referências}

1. King H, Aubert RE, Herman WL. Global burden of diabetes, 1995-2025. Diabetes Care 1998; 21:1414-31.

2. Wild S, Roglic G, Green A. Global prevalence of diabetes. Diabetes Care 2004; 27:1047-53.

3. Narayan KM, Gregg EW, Fagot-Campagna A, Engelgau MM, Vinicor F. Diabetes: a common, growing, serious, costly, and potentially preventable public health problem. Diabetes Res Clin Pract 2000; 50 Suppl 2:S77-84.

4. Davies MJ, Tringham JR, Troughton J, Khunti KK. Prevention of type 2 diabetes mellitus: a review of the evidence and its application in a UK setting. Diabet Med 2004; 21:403-14.

5. Unwin N, Shaw J, Zimmet P, Alberti KG. Impaired glucose tolerance and impaired fasting glycaemia: the current status on definition and intervention. Diabet Med 2002; 19:708-23.

6. Malerbi DA, Franco LJ. Multicenter study of the prevalence of diabetes mellitus and impaired glucose tolerance in the urban Brazilian population aged 30-69 yr. The Brazilian Cooperative Group on the Study of Diabetes Prevalence. Diabetes Care 1992; 15:1509-16.

7. Rosa RS, Schimidt MI, Duncan BB, Souza MFM, Lima AK, Moura L. Internações por diabetes mellitus como diagnóstico principal na rede pública do Brasil, 1999-2001. Rev Bras Epidemiol 2007; 10:465-78.

8. Barceló A, Aedo C, Rajpathak S, Robles S. The cost of diabetes in Latin America and the Caribbean. Bull World Health Organ 2003; 81:19-27.

9. Hu G, Jousilahti P, Tuomilehto J. Joint effects of history of hypertension at baseline and type 2 diabetes at baseline and during follow-up on the risk of coronary heart disease. Eur Heart J 2007; 28: 3059-66.

10. Nagpal J, Bhartia A. Cardiovascular risk profile of subjects with known diabetes from the middleand high-income group population of Delhi: the DEDICOM survey. Diabet Med 2008; 25:27-36.

11. Gorter PM, Visseren FLJ, Algra A, van der Graaf Y. The impact of site and extent of clinically evident cardiovascular disease and atherosclerotic burden on new cardiovascular events in patients with type 2 diabetes. The SMART Study. Diabet Med 2007; 24:1352-60.

12. Zhang X, Gregg EW, Cheng YJ, Thompson TJ, Geiss L, Duenas MR, et al. Diabetes mellitus and visual impairmemt. Arch Ophthalmol 2008; 126:1421-7.

13. Szklo M, Javier Nieto F. Epidemiology: beyond the basics. 2nd Ed. Sudbury: Jones \& Bartlett; 2007.

14. Silva NN. Amostragem probabilística: um curso introdutório. 2a Ed. São Paulo: Edusp; 2001.

15. World Health Organization. Report of a WHO Consultation. Definition, diagnosis and classification of diabetes mellitus and its complications. Part 1: diagnosis and classification of diabetes mellitus. Geneva: World Health Organization; 1999. (WHO/ NCD/NCS/99.2).
16. National Institutes of Health. Third Report of the National Cholesterol Education Program (NCEP) Expert Panel on Detection, Evaluation, and Treatment of High Blood Cholesterol in Adults (Adult Treatment Panel III). Final report. Bethesda: National Institutes of Health; 2002. (NIH Publication, 02-5215).

17. Habitch JP, Butz WP. Measurement of health and nutrition effects of large-scale nutrition intervention projects. In: Klein RE, editor. Evaluation of the impact of nutrition and health programs. New York: Plenum Press; 1979. p. 133-89.

18. Albert G, Zimmet P, Shaw J, Grundy SM. Metabolic syndrome. The IDF consensus worldwide definition of the metabolic syndrome. Brussels: International Diabetes Federation; 2006.

19. Matsudo S, Araújo T, Matsudo V, Andrade D, Andrade E, Oliveira LC, et al. Questionário Internacional de Atividade Física (IPAQ): estudo de validade e reprodutibilidade no Brasil. Rev Bras Ativ Fís Saúde 2001; 6:5-18.

20. Cohen JA. Coefficient of agreement for nominal scales. Educ Psychol 1960; 20:37-46.

21. Kleinbaum DG, Kupper LL, Müller KE, Nizam A. Applied regression analysis and other multivariable methods. 3rd Ed. Pacific Grove: Duxbury Press; 1998.

22. Castro V, Moraes SA, Mondini L, Freitas ICM. Variabilidade na aferição de medidas antropométricas: comparação de dois métodos estatísticos para avaliar a calibração de entrevistadores. Rev Bras Epidemiol 2008; 11:278-86.

23. Ministério da Saúde/Organização Pan-Americana da Saúde. Avaliação do plano de reorganização da atenção à hipertensão arterial e ao diabetes mellitus no Brasil. Brasília: Ministério da Saúde; 2004. (Série C. Projetos, Programas e Relatórios).

24. Ministério da Saúde. Portaria Conjunta no. 112, de 19 de junho de 2002. Diário Oficial da União 2002; 20 jun.

25. Torquato MTCG, Montenegro Júnior RM, Viana LAL, Souza RAHG, Lanna CMM, Lucas JCB, et al. Prevalence of diabetes mellitus and impaired glucose tolerance in urban population aged 30-69 years in Ribeirão Preto, SP, Brazil. São Paulo Med J 2003; 121:224-30.

26. Schaan BD, Harzheim E, Gus I. Perfil de risco cardíaco no diabetes mellitus e na glicemia de jejum alterada. Rev Saúde Pública 2004; 38:529-36.

27. Passos VMA, Barreto SM, Diniz LM, Lima-Costa MF Type 2 diabetes: prevalence and associated factors in a Brazilian community: the Bambuí Health and Aging Study. São Paulo Med J 2005; 123:66-71.

28. Martinez MC, Latorre MRDO. Fatores de risco para hipertensão arterial e diabetes mellitus em trabalhadores de empresa metalúrgica e siderúrgica. Arq Bras Cardiol 2006; 97:471-9.

29. Costa JSD, Olinto MTA, Assunção MCF, Gigante DP, Macedo S, Menezes AMB. Prevalência de diabetes mellitus em Pelotas, RS: um estudo de base populacional. Rev Saúde Pública 2006; 40:542-5. 
30. Felig P, Baxter JD, Broadus AE, Frohman LA. Endocrinology and metabolism. 2nd Ed. New York: McGrow-Hill Book Company; 1987.

31. Lee CMY, Huxley RR, Wildman RP, Woodward M. Indices of abdominal obesity are better discriminators of cardiovascular risk factors than BMI: a meta-analysis. J Clin Epidemiol 2008; 61:646-53.

32. Kagawa M, Byrne NM, Hills AP. Comparison of body fat estimation using waist:height ratio using different "waist" measurements in Australian adults. Br J Nutr 2008; 100:1135-41.

33. Gorallski KB, Sinal CJ. Type 2 diabetes and cardiovascular disease: getting to the fat of the matter. Can J Physiol Pharmacol 2007; 85:113-32.

34. Bener A, Zirie M, Janahi IM, Al-Hamaq AO, Musallam M, Wareham NJ. Prevalence of diagnosed and undiagnosed diabetes mellitus and its risk factors in a population-based study of Qatar. Diabetes Res Clin Pract 2009; 84:99-106.

35. Hadaegh F, Zabetian A, Harati H, Azizi F. Waist/ height ratio as a better predictor of tipe 2 diabetes compared to body mass index in Tehranian adult men-a 3.6 year prospective study. Exp Clin Endocrinol Diabetes 2006; 114:310-5.

36. Saaristo TE, Barengo NC, Korpi-Hyövälti E, Oksa H, Puolijoki H, Saltevo JT, et al. High prevalence obesity, central obesity and abnormal glucose tolerance in the middle aged Finnish population. BMC Public Health 2008; 8:423.
37. Nyamdorj R, Qiao Q, Söderberg S, Pitkäniemi JM, Zimmet PZ, Shaw JE, et al. BMI compared with central obesity indicators as a predictor of diabetes incidence in Mauritius. Obesity (Silver Spring) 2009; 17:342-8.

38. Hadaegh F, Shafiee G, Azizi F. Anthropometric predictors of incident type 2 diabetes mellitus in Iranian women. Ann Saudi Med 2009; 29:194-200.

39. He Y, Zhai F, Ma G, Feskens EJ, Zhang J, Fu P, et al. Abdominal obesity and the prevalence of diabetes and intermediate hyperglycaemia in Chinese adults. Public Health Nutr 20089; 12:1078-84.

40. Maty SC, Everson-Rose SA, Haan MN, Raghunathan TE, Kaplan GA. Education, income, occupation, and the 34-year incidence (1965-1999) of type 2 diabetes in the Alameda County Study. Int J Epidemiol 2005; 34:1274-81.

41. Maty SC, James SA, Kaplan GA. Life-course socioeconomic position and incidence of diabetes mellitus among blacks and whites: the Alameda County Study, 1965-1999. Am J Public Health 2010; 100:137-45.

Recebido em 29/Jun/2009

Versão final reapresentada em 23/Nov/2009

Aprovado em 11/Mar/2010 